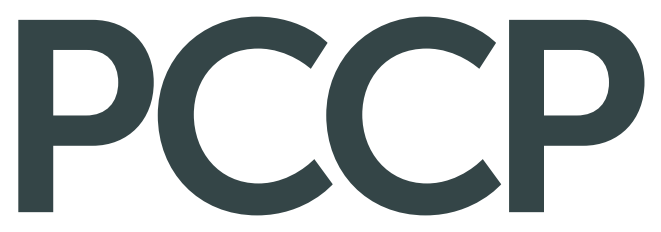

Physical Chemistry Chemical Physics www.rsc.org/pccp

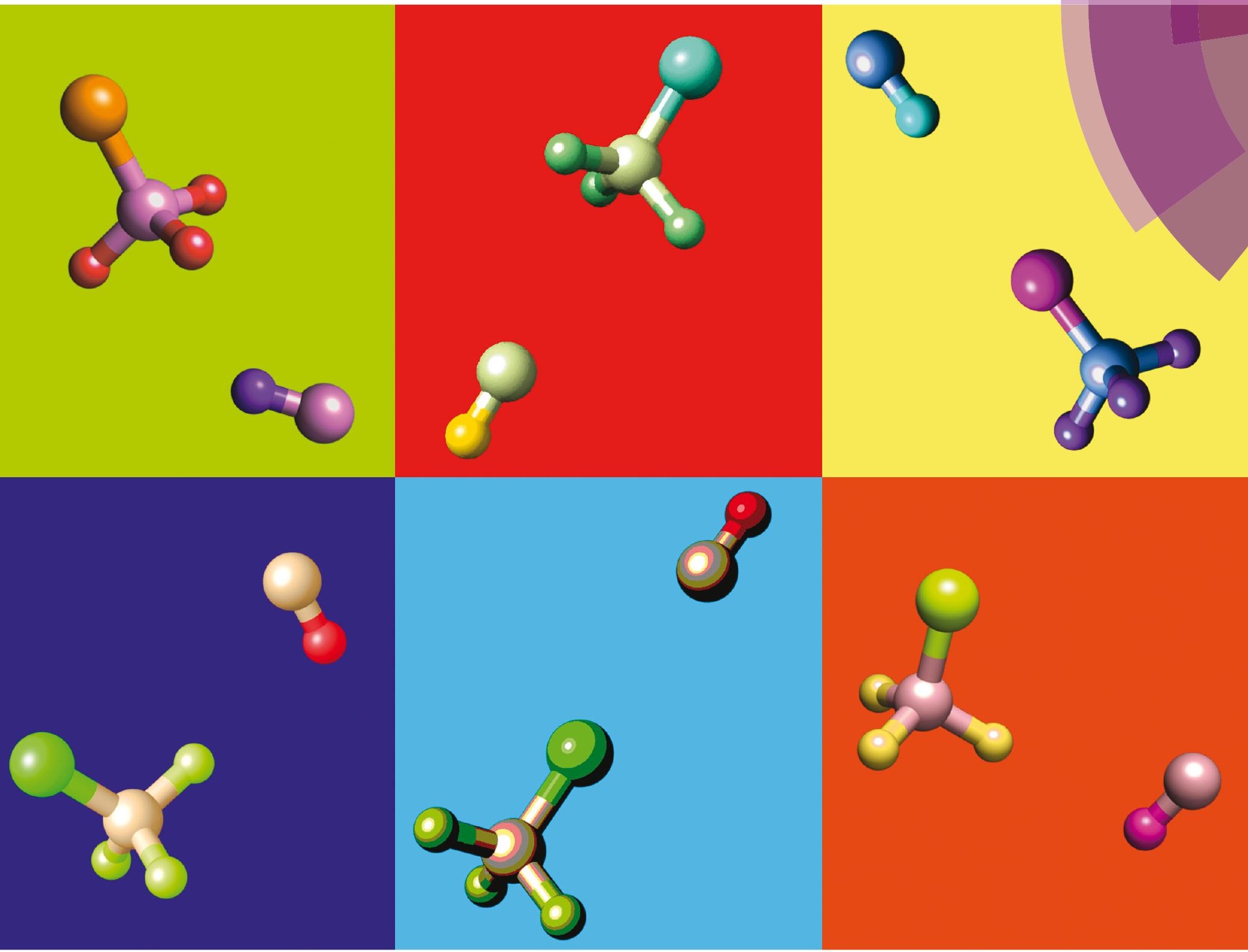

ISSN 1463-9076

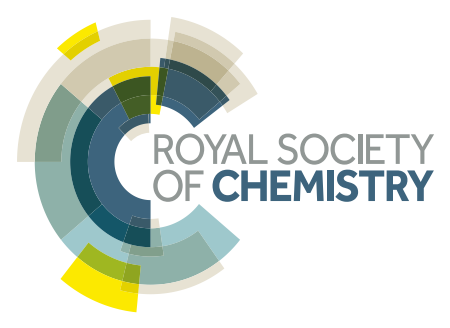




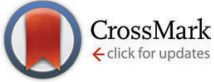

Cite this: Phys. Chem. Chem. Phys., 2016, 18, 17851

Received 16th February 2016, Accepted 13th March 2016

DOI: $10.1039 / c 6 c p 01059 h$

www.rsc.org/pccp

\section{On the $\mathrm{Cl}$... C halogen bond: a rotational study of $\mathrm{CF}_{3} \mathrm{Cl}-\mathrm{CO} \uparrow$}

\author{
Walther Caminati, ${ }^{a}{ }^{a}$ Luca Evangelisti, ${ }^{a}$ Gang Feng, ${ }^{a}$ Barbara M. Giuliano, ${ }^{a b}$ \\ Qian Gou, ${ }^{a}$ Sonia Melandri ${ }^{a}$ and Jens-Uwe Grabow ${ }^{c}$
}

\section{Introduction}

Many investigations are targeting the role of specific non-covalent interactions in different chemical and physical molecular systems. Most of these studies concern the hydrogen bond; however, several other interactions play important roles and together - cooperative or competitive - are crucial to determine the stable conformations of molecular systems.

Among them, halogen bonds (HaBs) and $n \rightarrow \pi^{*}$ are specific non-covalent interactions, which in some cases have been found to be competitive or preferred to the hydrogen bond (HB). Overviews on the $\mathrm{HaB}$ are available, ${ }^{1}$ and parallels with $\mathrm{HB}$ are reported. ${ }^{2}$ Its importance in supramolecular chemistry and crystal engineering has been outlined in several papers. ${ }^{3}$ Most of the investigations dedicated to the $\mathrm{HaB}$ employ X-ray diffraction and are relative to the solid state. ${ }^{4}$ The $n \rightarrow \pi^{*}$ (Bürgi-Dunitz) effect has been described in a number of molecular systems. ${ }^{5}$

In this paper we are interested in the characterization of the $\mathrm{HaB}$ interaction by means of the study of a molecular complex where the two subunits are held together through this kind of linkage. Some studies in this direction have been performed on halogen bonded complexes in cryo solutions using vibrational spectroscopy. ${ }^{6}$

Accurate details on the nature of the halogen bond in the gas phase can be obtained by rotational spectroscopy of molecular complexes, as shown in a review and perspective article by Legon. ${ }^{7}$

\footnotetext{
${ }^{a}$ Dipartimento di Chimica "G. Ciamician" dell'Università, Via Selmi 2,

I-40126 Bologna, Italy.E-mail: walther.caminati@unibo.it

${ }^{b}$ Max-Planck-Institut für extraterrestrische Physik, Giessenbachstrasse 1, 85748 Garching, Germany

Institut für Physikalische Chemie und Elektrochemie, Lehrgebiet A,

Gottfried-Wilhelm-Leibniz-Universität Callinstrasse 3A, D-30167 Hannover, Germany $\dagger$ Electronic supplementary information (ESI) available: Table of transition frequencies. See DOI: 10.1039/c6cp01059h
}

There, FTMW spectroscopy studies on a variety of B $\cdots \mathrm{XY}$ complexes, where $\mathrm{B}$ is the electron donor and $\mathrm{XY}$ is the dihalogen molecule, are reviewed to reveal some general properties of the halogen bond interaction. For example, information on the radial and angular geometries, intermolecular stretching forces and the extent of charge redistribution upon formation of the halogen bond has been reported. ${ }^{8}$ These studies also proved that the halogen bond is generally more linear than the hydrogen bond, with $\mathrm{B} \cdot \mathrm{X}-\mathrm{Y}$ angles very close to $180^{\circ}$.

We recently characterized the $\mathrm{HaB}$ interactions $(\mathrm{Cl} \cdots \mathrm{O}, \mathrm{Cl} \cdots \mathrm{N}$, and $\mathrm{Cl} \cdots \mathrm{F}$ ), in some complexes of chlorofluorocarbons (CFCs) with some small molecules. ${ }^{9}$ CFCs have a high impact on several atmospheric processes. Not only do CFCs have an important role in the greenhouse effect, but also they are involved in the depletion of ozone. Their atmospheric chemistry is rather complicated as complexation with other atmospheric molecules, such as CO, can affect their reactivity. Several experiments were performed on adducts of $\mathrm{ICF}_{3}$ with small molecules $\left(\mathrm{H}_{2} \mathrm{O}, \mathrm{NH}_{3}, \mathrm{CO}\right.$, etc. $){ }^{10}$ The final aim is to determine and establish general rules with predictive power toward the formation and properties of, in this case, halogen bonds. For this reason, we decided to investigate the rotational spectrum of the complex $\mathrm{CF}_{3} \mathrm{Cl}-\mathrm{CO}$. Here, we will give an answer to the following question: Which kind of $\mathrm{HaB}$ is preferred: a $\mathrm{Cl} \cdots \mathrm{C}$ or a $\mathrm{Cl} \cdots \mathrm{O}$ contact?

The spectrum was observed using a newly built supersonic-jet Fourier Transform microwave (FTMW) spectrometer in Bologna; its technical details are also reported. All experimental results are compared to standard ab initio theoretical calculations.

\section{Experimental section}

The spectrum has been recorded using the newly built supersonicjet Fourier-transform ${ }^{\mathbf{1 1}}$ microwave spectrometer of COBRA-type (coaxially oriented beam-resonator arrangement) in Bologna. ${ }^{12}$ 
We note that, while the implementation of this spectrometer has started ten years ago, the operation was locked-down for several years since the historic building of the Bologna Chemistry Department is under the tutelage of the fine arts, thus being subject to obtaining permission for the required installation of the pumping system's exhaust. Finally, after resolving this obstacle, we could collect, and report here, the first spectra of this instrument. It is designed to be combined with laser ablation and electrical discharge devices.

The spectrometer is based on an NI PXI-1042 chassis that is controlled by an NI PXI-8108 computer including a GPIBinterface and implementing Hannover FTMW++ software. It hosts the following data acquisition, timing, analog and digital I/O and motion control boards, respectively: (i) a NI PXI-5102 digital oscilloscope; (ii) a NI PXI-6602 timing I/O; (iii) a NI PXI6221 multifunction DAQ; (iv) a NI PXI-7344 motion controller supplemented with an NI MID-7604 4-axes stepper motor drive; (v) an LUH PXI-synchronization and an LUH experimentsequence-logic board built at the Leibniz Universität Hannover. Two subsequent down-conversion stages of $30 \mathrm{MHz}$ and 2.5 $\mathrm{MHz}$ are used to convert the molecular MW signal to an $\mathrm{RF}$ signal centered within the Nyquist range of the digitizer operated at a sampling rate of $10 \mathrm{MHz}$. The high-frequency electronic components (switches, amplifiers, a single-side-band modulator, an image-rejection mixer, band-pass and low-pass filters, and a complex (two-channel) detector) are similar to those of the Hannover FTMW spectrometer.

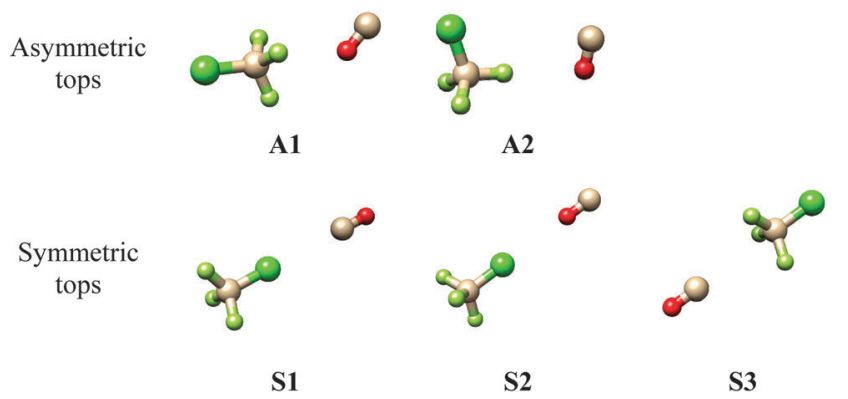

Fig. 1 Sketch of the two asymmetric top (An) and the three symmetric top (Sn) conformers of $\mathrm{CF}_{3} \mathrm{Cl}-\mathrm{CO}$.
The cavity, $50 \mathrm{~cm}$ diameter and $112 \mathrm{~cm}$ long, has been built by “Mori Meccanica srl", Parma. The vacuum is effectuated by interchangeable mechanical (rotary vane and roots booster) pumps at the foreline of a $12000 \mathrm{l} \mathrm{s}^{-1}$ oil-diffusion pump.

The supersonic-jet expansion is pulsed by a Series 9 solenoid valve which is controlled by an IOTA one valve driver governed by the experiment-sequence generation, both from the Parker Hannifin Corporation.

Molecular clusters were generated by supersonic expansion, under conditions optimized for the adduct formation. A mixture of $1 \% \mathrm{CO}$ and $1 \% \mathrm{CF}_{3} \mathrm{Cl}$ in helium at a stagnation pressure of $\sim 0.3 \mathrm{MPa}$ was expanded through a solenoid valve (nozzle orifice diameter $0.5 \mathrm{~mm}$ ) into the Fabry-Pérot type resonator. The spectral line positions were determined after Fourier transformation of the time-domain signal with $8 \mathrm{k}$ data points, sampled at $100 \mathrm{~ns}$ intervals. Each rotational transition appears as a doublet due to the Doppler effect of radiation propagating with and counter-propagating to the jet. The line rest frequency is calculated as the arithmetic mean of its two Doppler components. The estimated accuracy of the frequency measurements is better than $3 \mathrm{kHz}$. Lines separated by more than $7 \mathrm{kHz}$ are resolvable.

\section{Theoretical calculations}

Prior to recording of the microwave spectra, the properties of several conformations of the $\mathrm{CF}_{3} \mathrm{Cl}-\mathrm{CO}$ complex were predicted in silico. These computations yielded the molecular structures (and thus their rotational constants), the dipole moment components along the principal axes, the nuclear quadrupole coupling constants of the $\mathrm{Cl}$ nucleus, and the relative energies of the conformers.

All $a b$ initio calculations were performed using the Gaussian09 software package. ${ }^{13}$ The nature of all stationary points was verified by subsequent harmonic frequency calculations. We found five energy minima within $300 \mathrm{~cm}^{-1}$ : two of them are asymmetric tops $(\mathbf{A} \boldsymbol{n})$ while three correspond to symmetric tops (Sn). The shapes of these five conformations are drawn as Fig. 1, while their spectroscopic parameters and their relative energies are listed in Table 1. MP2 and B3LYP-D3 methods have been used, both with the $6-311++G(d, p)$ basis set.

Table 1 MP2/B3LYP-D3 (basis set 6-311++G(d,p) in both cases) spectroscopic parameters of all isomers of $\mathrm{CF}_{3} \mathrm{Cl}-\mathrm{CO}$

\begin{tabular}{|c|c|c|c|c|c|}
\hline & A1 & A2 & S1 & S2 & S3 \\
\hline$A / \mathrm{MHz}$ & $5438 / 5434$ & $3288 / 3268$ & $5718 / 5668$ & $5714 / 5669$ & $5709 / 5667$ \\
\hline$B / \mathrm{MHz}$ & $974 / 959$ & $932 / 1096$ & $605 / 616$ & $669 / 676$ & $782 / 788$ \\
\hline$C / \mathrm{MHz}$ & $966 / 952$ & $832 / 960$ & $605 / 616$ & $669 / 676$ & $782 / 788$ \\
\hline$\mu_{\mathrm{a}}^{a} / \mathrm{D}$ & $0.8 / 0.5$ & $0.4 / 0.1$ & $1.1 / 0.8$ & $0.5 / 0.6$ & $0.5 / 0.3$ \\
\hline$\mu_{\mathrm{b}} / \mathrm{D}$ & $0.1 / 0.0_{3}$ & $0.3 / 0.4$ & $0.0 / 0.0$ & $0.0 / 0.0$ & $0.0 / 0.0$ \\
\hline$\chi_{\mathrm{aa}} / \mathrm{MHz}$ & $-72.2 /-75.6$ & $23.4 / 20.0$ & $-74.0 /-76.6$ & $-73.6 /-76.6$ & $-73.2 /-76.2$ \\
\hline$\chi_{\mathrm{bb}} / \mathrm{MHz}$ & $35.4 / 37.4$ & $-60.3 /-58.2$ & $37.0 / 38.3$ & $36.8 / 38.3$ & $36.6 / 38.1$ \\
\hline$\chi_{\mathrm{cc}} / \mathrm{MHz}$ & $36.8 / 38.2$ & $36.9 / 38.2$ & $37.0 / 38.3$ & $36.8 / 38.3$ & $36.6 / 38.1$ \\
\hline$\chi_{\mathrm{ab}} / \mathrm{MHz}$ & $12.3 / 9.6$ & $36.1 / 42.0$ & & & \\
\hline$E / \mathrm{cm}^{-1}$ & $0^{b} / 63$ & $62 / 125$ & $13 / 0^{c}$ & $84 / 96$ & $87 / 84$ \\
\hline$E_{0} / \mathrm{cm}^{-1}$ & $0^{d}$ & 39 & 20 & 80 & 80 \\
\hline$D_{\mathrm{e}} / \mathrm{kJ} \mathrm{mol}^{-1}$ & 4.8 & 4.0 & 4.6 & 3.8 & 3.8 \\
\hline
\end{tabular}




\section{Rotational spectrum and analysis}

The rotational spectrum of the most abundant isotopic species $\left({ }^{35} \mathrm{Cl}, \mathrm{ca} .75 \%\right.$ of natural abundance $)$ has been investigated first. It exhibited the spectrum of a symmetric-top molecule, with bands evenly separated by $2 B$. At the left part of each band (lower frequency), a weaker spectrum, belonging to the ${ }^{37} \mathrm{Cl}$ isotopologue ( $\mathrm{ca}$. 25\% of natural abundance) was also observed. The first assignments were relative to $K=0$ and \pm 1 transitions of the $J=7 \leftarrow 6$ band (shown in Fig. 2).

Each transition was split into several component lines, due to the nuclear quadrupole coupling of the $\mathrm{Cl}\left({ }^{35} \mathrm{Cl}\right.$ and ${ }^{37} \mathrm{Cl}$, $I=3 / 2$ ) nucleus. Moreover, due to coaxial geometry of the spectrometer, doubling of the signals was originated by the Doppler effect (see Experimental section). The fit was performed using Pickett's SPFIT program, ${ }^{14}$ according to the Hamiltonian:

$$
H=H_{\mathrm{R}}+H_{\mathrm{CD}}+H_{\mathrm{Q}}(\mathrm{Cl})
$$

in which $H_{\mathrm{R}}$ represents the rigid rotational part of a symmetric top Hamiltonian, $H_{\mathrm{CD}}$ takes into account the corresponding first order centrifugal distortion contributions and $H_{\mathrm{Q}}(\mathrm{Cl})$ includes the interaction of the ${ }^{35} \mathrm{Cl}\left(\right.$ or $\left.{ }^{37} \mathrm{Cl}\right)$ nuclear quadrupole moment with the electric field gradient at the $\mathrm{Cl}$ nucleus. Because of the symmetry of the complex, just one directional quadrupole coupling constant, $\chi_{\mathrm{aa}}$, is required to describe the three-dimensional field gradient (all measured lines are given in the ESI $\dagger$ ). The determined spectroscopic parameters are reported in Table 2.

No other intense transitions have been observed in the rotational spectrum. The MP2 ab initio calculations suggest rotamer A1 to be the most stable one, but, in agreement with the B3LYP-D3 predictions, we observed only a symmetric top spectrum. The experimental rotational constant $B$, as well as the quadrupole coupling constant $\chi_{\text {aa }}$, are close to the theoretical values of species S1, especially to the B3LYP-D3 values (616 and $-76.6 \mathrm{MHz}$ against the $\sim 621$ and $\sim-76.8 \mathrm{MHz}$ experimental values, respectively); then, most

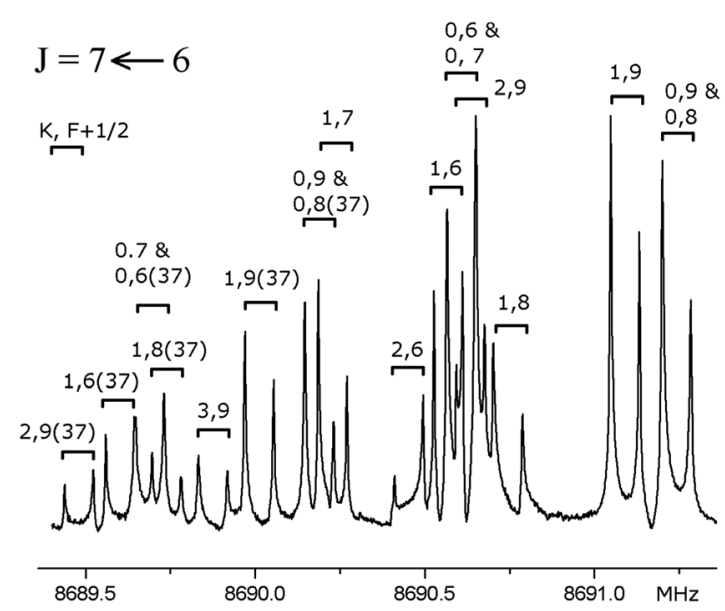

Fig. 2 Recorded $\mathrm{J}=7 \leftarrow 6$ band composed of ${ }^{35} \mathrm{Cl}$ and ${ }^{37} \mathrm{Cl}$ isotopologue transitions. (37) denotes transitions of the ${ }^{37} \mathrm{Cl}$ isotopologue. 26546 molecular time-domain signals were accumulated prior to Fourier transform. Each line appears as a doublet $(\sqcap)$ due to the Doppler effect.
Table 2 Experimental spectroscopic parameters of the observed (S1) conformer of $\mathrm{CF}_{3} \mathrm{Cl}-\mathrm{CO}$

\begin{tabular}{lll}
\hline & ${ }^{35} \mathrm{Cl}$ & ${ }^{37} \mathrm{Cl}$ \\
\hline$B / \mathrm{MHz}$ & $620.8354(1)^{a}$ & $620.7636(2)$ \\
$\chi_{\mathrm{aa}} / \mathrm{MHz}$ & $-76.773(1)$ & $-60.77(1)$ \\
$D_{\mathrm{J}} / \mathrm{kHz}$ & $0.4989(4)$ & $0.4971(9)$ \\
$D_{\mathrm{JK}} / \mathrm{kHz}$ & $19.78(1)$ & $19.7(1)$ \\
$N^{b}$ & 80 & 46 \\
$\sigma^{c} / \mathrm{kHz}$ & 1 & 2
\end{tabular}

${ }^{a}$ Error in parenthesis is expressed in units of the last digit. ${ }^{b}$ Number of lines in the fit. ${ }^{c}$ Standard deviation of the fit.

probably this is the observed rotamer. The calculated energy difference between $\mathbf{A 1}$ and $\mathbf{S 1}$ is relatively small, reasonably below the accuracy of the chosen theoretical methods. Although A1, at the reliability of the theoretical calculations, might only be slightly higher in energy than S1, it is not surprising that its spectrum is not observed (as well as those of the other low energy rotamers) because effective conformational relaxation to the most stable conformers takes place, upon supersonic expansion, at low interconversion barriers. ${ }^{15}$

\section{Location of the $\mathrm{Cl}$ atom in the complex}

From the rotational constants $B$ of the ${ }^{35} \mathrm{Cl}$ and ${ }^{37} \mathrm{Cl}$ species it was possible to calculate the substitution $a$-coordinate (see Table 3) of the $\mathrm{Cl}$ atom in the principal axes system of the parent species, according to Kraitchman's equations. ${ }^{16}$ Its value constitutes further evidence supporting the assignment of the observed spectrum to rotamer S1, with the discrepancies between experimental and theoretical values being $c a$. 0.02 and $0.10 \AA$ for $\mathbf{S 1}$ and $\mathbf{S} 2$, respectively.

\section{Dissociation energy}

The stretching motion which leads to dissociation takes place along the $a$-symmetry axis. Assuming this motion to be isolated from the other (bending) motions, the stretching force constant $\left(k_{\mathrm{s}}\right)$ can be estimated by approximating the complex to be a 'pseudodiatomic molecule' made from two rigid parts, according to: ${ }^{17}$

$$
k_{\mathrm{S}}=\left(16 \cdot \pi^{2} \cdot \mu \cdot B_{\mathrm{D}}{ }^{3} / D_{\mathrm{J}}\right) \cdot\left(1-B_{\mathrm{D}} / B_{\mathrm{CFC}}-B_{\mathrm{D}} / B_{\mathrm{B}}\right)
$$

where $\mu$, and $D_{\mathrm{J}}$ are the "pseudo diatomic" reduced mass and the first-order centrifugal distortion constants. $B_{\mathrm{D}}, B_{\mathrm{CFC}}$ and $B_{\mathrm{B}}$ are the rotational constants of the dimer and the two monomers, respectively. A $k_{\mathrm{s}}$ value of $2.2 \mathrm{~N} \mathrm{~m}^{-1}$, corresponding to a stretching frequency of $42 \mathrm{~cm}^{-1}$, was obtained. This equation

Table 3 Comparison of the experimental $\left(r_{\mathrm{s}}\right)$ a-coordinate of the $\mathrm{Cl}$ atom to the theoretical $\left(r_{\mathrm{e}}\right)$ values for the symmetric species $\mathbf{S} \mathbf{1}$ and $\mathbf{S 2}$

\begin{tabular}{lll}
\hline & $a(\mathrm{Cl}) / \AA$ & \\
\hline$r_{\mathrm{s}}$ (expt) & & $\pm 0.219(7)^{a}$ \\
$r_{\mathrm{e}}$ (theor.) & S1 & 0.241 \\
& S2 & 0.312
\end{tabular}

${ }^{a}$ Error in parenthesis is expressed in units of the last digit. 


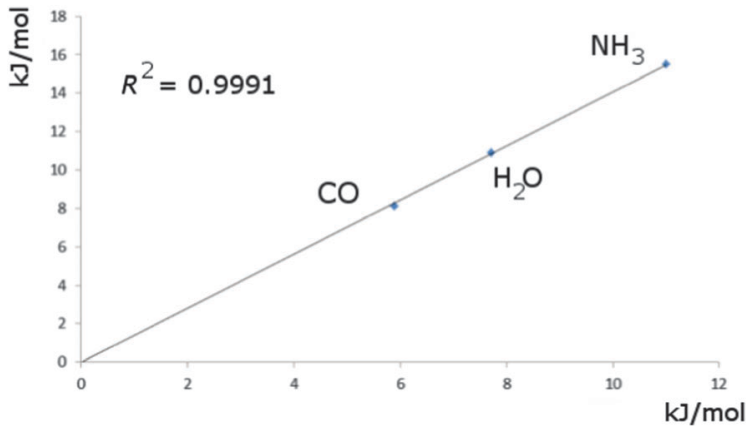

Fig. 3 Relationship of the $E_{\mathrm{D}}$ dissociation energy of $\mathrm{B} \ldots \mid \mathrm{CF}_{3}$ (vertical axis) versus that of $\mathrm{B} \cdots \mathrm{ClCF}_{3}$ (horizontal axis). While the value of $R^{2}$ is essentially 1 , it should still be taken with caution because of the size of the dataset.

has the advantage of allowing the calculation of $k_{\mathrm{s}}$ directly from the centrifugal distortion constants and rotational constants without requiring the evaluation of any structural parameter of the dimer. By assuming a Lennard-Jones potential function and using the approximated equation: ${ }^{18}$

$$
E_{\mathrm{D}}=k_{\mathrm{s}} \cdot R_{\mathrm{CM}}^{2} / 72
$$

where $R_{\mathrm{CM}}$ is the distance between the two centers of mass. The dissociation energy $\left(E_{\mathrm{D}}\right)$ was evaluated to be $5.9 \mathrm{~kJ} \mathrm{~mol}^{-1}$.

Since several halogen bonded adducts (B ‥CFC) of CFCs with small partner molecules (B) have been investigated, it is possible to establish empirical trends and relationships for this kind of intermolecular interaction.

In Fig. 3, the $E_{\mathrm{D}}$ values of the $\mathrm{B} \cdots \mathrm{ICF}_{3}$ adducts are plotted versus the $E_{\mathrm{D}}$ values of the $\mathrm{B} \cdots \mathrm{ClCF}_{3}$ adducts for $\mathrm{B}=\mathrm{CO}, \mathrm{H}_{2} \mathrm{O}$ and $\mathrm{NH}_{3}$. The least-squares fit of the points, with a coefficient of determination $R^{2} \sim 0.9991$ (very close to 1 ), leads to a slope of 1.4. This indicates that the $\mathrm{B} \cdots \mathrm{ICF}_{3}$ linkage is systematically

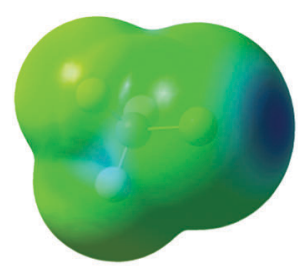

$\mathrm{CF}_{3} \mathrm{Cl}$

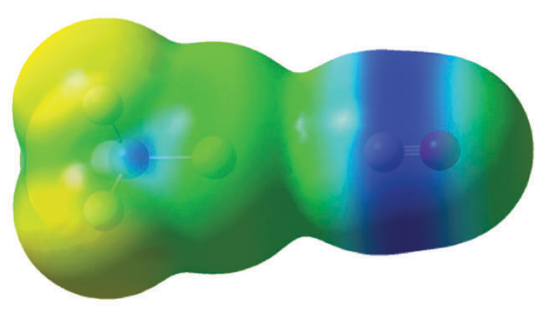

$\mathrm{CF}_{3} \mathrm{Cl}-\mathrm{CO}$

Fig. $4 A b$ initio molecular electrostatic potential surface plotted on the van der Waals surface of the $\mathrm{CF}_{3} \mathrm{Cl}$ and $\mathrm{CO}$ monomers and their complex. The potential ranges from $\sim-10^{-2} \mathrm{~V}$ (red) to $\sim 10^{-2} \mathrm{~V}$ (blue). stronger than the $\mathrm{B} \cdot \mathrm{ClCF}_{3}$ contact. The experimental evidence is that heavier halogens in CFCs form stronger halogen bonds.

To better understand the underlying interactions that govern the observed conformers, a molecular electrostatic potential surface (ESP) analysis was performed using Gaussian09. In general, molecular ESP maps can be used to (i) identify electron-rich and electron-deficient sites of various molecules, (ii) analyze the influence of conformation on those sites, and (iii) shed light on the inter-molecular bonding interactions occurring in the system. The ESP surfaces of the monomers and complex I are graphically reported in Fig. 4.

The chlorine atom displays a small positive ESP cap on the outer side of $\mathrm{X}$ along the extension of the $\mathrm{C}-\mathrm{X}$ bond, which primarily accounts for the directionality of the halogen bond $\mathrm{HaB}$. Instead a large region of negative ESP is centered on the carbon atom for CO.

\section{Conclusion}

In this article, the design and performance of a new molecular beam FTMW spectrometer is reported. The measurement of the rotational spectrum of $\mathrm{CF}_{3} \mathrm{Cl}-\mathrm{CO}$ demonstrates the sensitivity and the relative intensity accuracy of this spectrometer. The experimental configuration of the complex is characterized by a halogen bond interaction. The position of the chlorine atom was evaluated, together with the force constant, vibrational frequency, and dissociation energy of the complex. The energy of this interaction is similar to that of a weak hydrogen bond. All these data have been obtained for the first time with a high resolution spectroscopic technique. In addition, experimental evidence suggests that, at least for this type of complex, the theoretical B3LYP-D3 method performs better than the MP2 one.

\section{Acknowledgements}

We thank the Italian MIUR (PRIN projects 2004033958_003, 2008KJX4SN_001, and 2010ERFKXL_001) and the University of Bologna (RFO) for financial support. L. E. was supported by a Marie Curie fellowship PIOF-GA-2012-328405. G. F. and Q. G. also thank the China Scholarship Council (CSC) for financial support. J.-U. G. is indebted to the Deutsche Forschungsgemeinschaft (DFG) and the Land Niedersachsen for financial support.

\section{Notes and references}

1 P. Politzer, P. Lane, M. C. Concha, Y. Ma and J. S. Murray, J. Mol. Model., 2007, 13, 305-311.

2 See, for example, P. Metrangolo, H. Neukirch, T. Pilati and G. Resnati, Acc. Chem. Res., 2005, 38, 386; P. Metrangolo and G. Resnati, Science, 2008, 321, 918, and references therein.

3 See, for example, P. Metrangolo, G. Resnati, T. Pilati, R. Liantonio and F. Meyer, J. Polym. Sci., Part A: Polym. Chem., 2007, 45, 1, and references therein. 
4 See, for example, D. Braga, G. R. Desiraju, J. S. Miller, A. Guy Orpen and S. L. Price, CrystEngComm, 2002, 4, 500-509, and references therein.

5 M. E. Sanz, A. Lesarri, M. I. Peña, V. Vaquero, V. Cortijo, J. C. López and J. L. Alonso, J. Am. Chem. Soc., 2006, 128, 3812; S. Blanco, J. C. López, S. Mata and J. L. Alonso, Angew. Chem., Int. Ed., 2010, 49, 9187; Q. Gou, G. Feng, L. Evangelisti and W. Caminati, Angew. Chem., Int. Ed., 2013, 52, 11888; Q. Gou, L. Spada, Y. Geboes, W. A. Herrebout, S. Melandri and W. Caminati, Phys. Chem. Chem. Phys., 2015, 17, 7694.

6 D. Hauchecorne, B. J. van der Veken, A. Moiana and W. Herrebout, Chem. Phys., 2010, 374, 30-36; D. Hauchecorne, R. Szostak, W. A. Herrebout and B. J. van der Veken, ChemPhysChem, 2009, 10, 2105.

7 A. C. Legon, Angew. Chem., Int. Ed., 1999, 38, 2686.

8 A. C. Legon, Phys. Chem. Chem. Phys., 2010, 12, 7736.

9 L. Evangelisti, G. Feng, P. Écija, E. J. Cocinero, F. Castaño and W. Caminati, Angew. Chem., Int. Ed., 2011, 50, 7807; G. Feng, L. Evangelisti, N. Gasparini and W. Caminati, Chem. - Eur. J., 2012, 18, 1364; L. Evangelisti, G. Feng, Q. Gou, J.-U. Grabow and W. Caminati, J. Phys. Chem. A, 2014, 118, 579; Q. Gou, L. Spada, E. J. Cocinero and W. Caminati, J. Phys. Chem. Lett., 2014, 5, 1591.

10 S. L. Stephens, N. R. Walker and A. C. Legon, J. Chem. Phys., 2011, 135, 224309; S. L. Stephens, N. R. Walker and A. C. Legon, Phys. Chem. Chem. Phys., 2011, 13, 21093; S. L. Stephens, N. R. Walker and A. C. Legon, Phys. Chem. Chem. Phys., 2011, 13, 20736.

11 T. J. Balle and W. H. Flygare, Rev. Sci. Instrum., 1981, 52, 33.
12 J.-U. Grabow and W. Stahl, Z. Naturforsch., A: Phys. Sci., 1990, 45, 1043; J.-U. Grabow, Doctoral thesis, ChristianAlbrechts-Universität zu Kiel, Kiel, 1992; J.-U. Grabow, W. Stahl and H. Dreizler, Rev. Sci. Instrum., 1996, 67, 4072; J.-U. Grabow, Habilitationsschrift, Universität Hannover, Hannover, 2004, program available at http://www.pci.unihannover.de/ lgpca/spectroscopy/ftmw.

13 M. J. Frisch, G. W. Trucks, H. B. Schlegel, G. E. Scuseria, M. A. Robb, J. R. Cheeseman, G. Scalmani, V. Barone, B. Mennucci, G. A. Petersson, H. Nakatsuji, M. Caricato, X. Li, H. P. Hratchian, A. F. Izmaylov, J. Bloino, G. Zheng, J. L. Sonnenberg, M. Hada, M. Ehara, K. Toyota, R. Fukuda, J. Hasegawa, M. Ishida, T. Nakajima, Y. Honda, O. Kitao, H. Nakai, T. Vreven, J. A. Montgomery, Jr., J. E. Peralta, F. Ogliaro, M. Bearpark, J. J. Heyd, E. Brothers, K. N. Kudin, V. N. Staroverov, R. Kobayashi, J. Normand, K. Raghavachari, A. Rendell, J. C. Burant, S. S. Iyengar, J. Tomasi, M. Cossi, N. Rega, J. M. Millam, M. Klene, J. E. Knox, J. B. Cross, V. Bakken, C. Adamo, J. Jaramillo, R. Gomperts, R. E. Stratmann, O. Yazyev, A. J. Austin, R. Cammi, C. Pomelli, J. W. Ochterski, R. L. Martin, K. Morokuma, V. G. Zakrzewski, G. A. Voth, P. Salvador, J. J. Dannenberg, S. Dapprich, A. D. Daniels, Ö. Farkas, J. B. Foresman, J. V. Ortiz, J. Cioslowski and D. J. Fox, Gaussian, Inc., Wallingford CT, 2009.

14 H. M. Pickett, J. Mol. Spectrosc., 1991, 148, 371.

15 See for example: R. S. Ruoff, T. D. Klots, T. Emilson and H. S. Gutowski, J. Chem. Phys., 1990, 93, 3142.

16 J. Kraitchman, Am. J. Phys., 1953, 21, 17.

17 D. J. Millen, Can. J. Chem., 1985, 63, 1477; W. G. Read, E. J. Campbell and G. Henderson, J. Chem. Phys., 1983, 78, 3501.

18 S. E. Novick, S. J. Harris, K. C. Janda and W. Klemperer, Can. J. Phys., 1975, 53, 2007. 